\title{
Dynamical Casimir Emission from Polariton Condensates
}

\author{
Selma Koghee* and Michiel Wouters \\ TQC, Universiteit Antwerpen, Universiteitsplein 1, B-2610 Wilrijk, Belgium
}

(Received 5 March 2013; published 24 January 2014)

\begin{abstract}
We study theoretically the dynamical Casimir effect in an exciton-polariton condensate that is suddenly created by an ultrashort laser pulse at normal incidence. As a consequence of the abrupt change of the quantum vacuum, Bogoliubov excitations are generated. The subsequent evolution, governed by polariton interactions and losses, is studied within a linearized truncated Wigner approximation. We focus in particular on the momentum distribution and spatial coherence. The limiting behavior at large and small momenta is determined analytically. A simple scaling relation for the final condensate depletion as a function of the system parameters is found and the correlation length is shown to depend linearly on the condensate depletion.
\end{abstract}

DOI: 10.1103/PhysRevLett.112.036406

PACS numbers: 71.36.+c, 03.75.Kk, 42.25.Kb, 42.50.-p

Interacting systems possess a rich ground-state structure, as a profound consequence of quantum mechanics. An immediate implication is the dynamical Casimir effect: when some parameter is abruptly varied, the system does not find itself in the new ground state, causing excitations to appear. Since the original idea of generating photons by accelerating two mirrors with respect to each other $[1,2]$, the dynamical Casimir effect has been studied in contexts as diverse as particle production in a time dependent cosmological background [3-5], superconducting circuits [6,7], and ultracold atomic gases [8-11]. In the latter system, the nontrivial vacuum is that of the (weakly) interacting Bose gas, which features quantum depletion.

A different realization of the weakly interacting Bose gas is formed by exciton polaritons in a microcavity [12]. Their advantage over atomic gases is that exciton polaritons contain a photonic component, offering great flexibility for optically creating them in a specific state. This feature was, e.g., exploited in the experiments on polariton superfluidity, where the polariton velocity could be varied by simply changing the angle of incidence of the excitation laser [13].

This advantage has recently led to several proposals to utilize exciton polaritons for the experimental observation of Hawking radiation $[14,15]$. In this Letter, we analyze the dynamical Casimir effect, a phenomenon intimately related to Hawking radiation. We propose to create polaritons by a pulsed laser at normal incidence, which results in a state with all particles at zero momentum. This is not the true ground state of the interacting many-body system, where the condensate is depleted as a fraction of the particles has nonzero momentum. Therefore, quasiparticles are expected to appear. In other terms, by the sudden creation of a coherent polariton population, the vacuum is changed from the trivial one to the Bogoliubov vacuum, leading to dynamical Casimir emission, see Fig. 1. The change of the dispersion due to the presence of a condensate was probed in the experiments of Kohnle et al. [16] by a four-wave-mixing pump-probe experiment. We suggest here to study the dynamics without the probe pulse.

An important aspect of polariton physics in actual experiments is the finite polariton lifetime. This brings the system in an inherently out-of-equilibrium situation and limits the possibilities to observe quantum effects. On the other hand, it offers a unique laboratory to study the interplay and competition between quantum correlations and dissipation, which may teach us lessons that are relevant for cosmological studies [17]. A basic question is how the finite polariton lifetime affects the dynamical Casimir generation of excitations. We will show that within the Bogoliubov approximation, the dynamical quantum depletion of a one-dimensional polariton wire [18] scales linearly with a "blockade" parameter $U / \gamma$ [19], where $\gamma$ is the homogeneous linewidth and $U$ is the interaction energy of two polaritons that are localized within the condensate healing length.

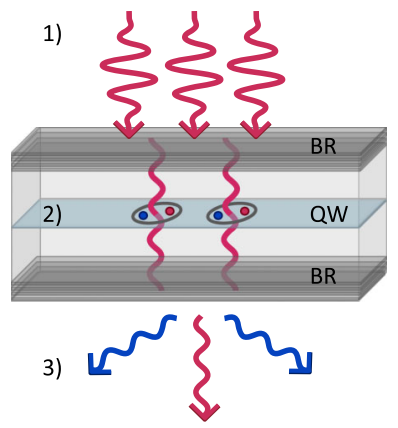

FIG. 1 (color online). When a short laser pulse (1) is shone onto a (quasi-one-dimensional) microcavity, photons become confined between the two Bragg reflectors (BR). The laser frequency is chosen to be resonant with an excitation in the quantum well (QW). This results in the creation of a polariton condensate (2) and the trivial vacuum suddenly changes to the Bogoliubov one. Consequently, dynamical Casimir emission of Bogoliubov excitations is generated (3). 
The coupling between the polariton fluid and the outside world leads both to particle losses and to the entrance of vacuum fluctuations into the system. This coupling can be described accurately with the master equation [20]. An equivalent formulation can be constructed in terms of the Wigner distribution of the polariton field [20]. This approach comes down to mapping the quantum master equation to a set of classical differential equations for stochastic fields that sample the Wigner distribution function. The stochastic differential equation for the polariton field $\varphi(\mathbf{x}, t)$ reads in the so-called truncated Wigner approximation [21]

$$
\begin{aligned}
i \hbar d \varphi(\mathbf{x}, t)= & {\left[\epsilon(-i \vec{\nabla})-i \frac{\gamma}{2}+g|\varphi(\mathbf{x}, t)|^{2}\right] \varphi(\mathbf{x}, t) d t } \\
& +\sqrt{\frac{\hbar \gamma}{4 \Delta V}} d W(\mathbf{x}, t),
\end{aligned}
$$

where $\epsilon(-i \vec{\nabla})$ gives the kinetic energy of a free particle, $g$ is the strength of the contact interaction, and $\Delta V$ is the volume of the grid cell necessary in the derivation of the truncated Wigner approximation. The stochastic term $d W$ is white Gaussian noise with amplitude variance $\left\langle d W^{*}(\mathbf{x}, t) d W\left(\mathbf{x}^{\prime}, t\right)\right\rangle=2 d t \delta_{\mathbf{x}, \mathbf{x}^{\prime}}$ and a random phase. Expectation values with respect to the Wigner distribution of the stochastic fields correspond to the symmetric operator averages, e.g., $\left\langle\varphi^{*}(\mathbf{x}, t) \varphi\left(\mathbf{x}^{\prime}, t\right)\right\rangle_{W}=1 / 2\left\langle\hat{\psi}^{\dagger}(\mathbf{x}, t) \times\right.$ $\left.\hat{\psi}\left(\mathbf{x}^{\prime}, t\right)+\hat{\psi}\left(\mathbf{x}^{\prime}, t\right) \hat{\psi}^{\dagger}(\mathbf{x}, t)\right\rangle$. This allows for a direct computation of all equal time correlation functions [20]. Although the mapping between the quantum and classical problem becomes exact only in the case of a quadratic Hamiltonian [20], the truncated Wigner formulation does not introduce any further errors in our calculations, since our computations are restricted to the Bogoliubov approximation.

The action of a resonant excitation pulse at $\mathbf{k}=0$ can be included in Eq. (1) by adding a pumping term $F_{L}(\mathbf{x}, t)$ on the right-hand side. However, for a very short laser pulse, the pumping term can be replaced by a shift of the initial condition of the stochastic variable

$$
\varphi(\mathbf{x}, t=0)=\eta(\mathbf{x})+\int F_{L}\left(t^{\prime}\right) e^{i \epsilon(0) t^{\prime} / \hbar} d t^{\prime},
$$

where $\eta(\mathbf{x})$ is the normally distributed uncorrelated vacuum noise with variance $\left\langle\eta^{*}(\mathbf{x}) \eta\left(\mathbf{x}^{\prime}\right)\right\rangle_{W}=1 / 2 \delta_{\mathbf{x}, \mathbf{x}^{\prime}}$. The subsequent dynamics are no longer influenced by the exciting laser. This situation is different from both resonant and nonresonant continuous wave excitation schemes, where an interplay between driving and dissipation takes place at all times. Compared to the continuous wave excitation regime, the dynamics of the polariton gas in the present scheme resembles the standard Bose gas more closely.

For the zero pulse width approximation in Eq. (2) to be valid, the pulse duration $\tau_{L}$ should be shorter than $\hbar /\left[g n_{c}(0)\right]$, where $n_{c}(0)$ is the initial condensate density, since the blueshift $g n_{c}(0)$ is the largest energy scale for polaritons with small momenta. At the same time, $\tau_{L}$ should be longer than the Rabi period $\Omega_{R}^{-1}$, in order to prevent creating polaritons in the upper branch. Thus, for typical values $g n_{c}(0)=0.5 \mathrm{meV}$ and $\Omega_{R}=10 \mathrm{meV}$, pulse duration is restricted to $66 \mathrm{fs} \ll \tau_{L} \ll 1.3 \mathrm{ps}$. Although the frequency of the laser does not play a crucial role in the discussed excitation scheme, the best efficiency will be obtained for the frequency corresponding to the blue shifted polariton energy.

In our calculations, the polariton field is decomposed into a condensate $(\mathbf{k}=0)$ and fluctuation $(\mathbf{k} \neq 0)$ component: $\varphi(\mathbf{x}, t)=\varphi_{c}(t)+\delta \varphi(\mathbf{x}, t)$, where $\left|\varphi_{c}(t)\right| \gg|\delta \varphi(\mathbf{x}, t)|$. Solving Eq. (1) for the condensate component, i.e., without the fluctuations and the noise term, yields

$$
\varphi_{c}(t)=\sqrt{n_{c}(t)} \exp \left[-i \theta_{c}(t)\right],
$$

where the condensate density $\left\langle\varphi_{c}^{*}(\mathbf{x}, t) \varphi_{c}(\mathbf{x}, t)\right\rangle_{W} \equiv$ $n_{c}(t)=n_{c}(0) e^{-\gamma t / \hbar}$ decays exponentially and its phase is given by the time integrated blueshift $\theta_{c}(t)=$ $g / \hbar \int_{0}^{t} n_{c}\left(t^{\prime}\right) d t^{\prime}$. Up to first order, the motion of the fluctuations is governed by the familiar Bogoliubov matrix

$$
B_{\mathbf{k}}(t)=\left(\begin{array}{cc}
\epsilon(k)+g n_{c}(t)-i \gamma / 2 & g n_{c}(t) \\
-g n_{c}(t) & -\epsilon(k)-g n_{c}(t)-i \gamma / 2
\end{array}\right)
$$

as

$$
i \hbar d \Phi_{\mathbf{k}}=B_{\mathbf{k}}(t) \Phi_{\mathbf{k}} d t+\frac{\sqrt{\hbar \gamma}}{2} d \Xi_{\mathbf{k}}(t),
$$

where $\quad \Phi_{\mathbf{k}}=\left[e^{-i \theta_{c}} \delta \varphi(\mathbf{k}) e^{i \theta_{c}} \delta \varphi^{*}(-\mathbf{k})\right]^{T} \quad$ and $\quad d \Xi_{\mathbf{k}}(t)=$ $\left[d W(\mathbf{k}, t)-d W^{*}(-\mathbf{k}, t)\right]^{T}$, with the correlation in momentum space $\left\langle d W^{*}(\mathbf{k}, t) d W\left(\mathbf{k}^{\prime}, t\right)\right\rangle=2 d t \delta_{\mathbf{k}, \mathbf{k}^{\prime}}$.

Because of the time dependence of the Bogoliubov matrix, an analytic solution for the fluctuations $\Phi_{\mathbf{k}}$ is not straightforward to compute, but for small and large wave vectors, limiting expressions can be found. Let us start with the limit $\mathbf{k} \rightarrow 0$. In this case, the time dependence of the real part of the Bogoliubov matrix comes down to a rescaling with a factor $\exp (-\gamma t / \hbar)$, whereas the imaginary part is proportional to the identity matrix. Hence, the Bogoliubov matrices at different times commute with each other. The differential equations (5) are then easily solved, yielding for the momentum distribution

$$
\begin{aligned}
\lim _{k \rightarrow 0} n(\mathbf{k}, t) & =\lim _{k \rightarrow 0}\left\langle\delta \varphi^{*}(\mathbf{k}) \delta \varphi(\mathbf{k})\right\rangle_{W}-\frac{1}{2} \\
& =2\left(\frac{g n_{c}(0)}{\gamma}\right)^{2} e^{-2 \gamma t / \hbar}\left(e^{\gamma t / \hbar}-\frac{\gamma t}{\hbar}-1\right),
\end{aligned}
$$

where we have used the relation between the momentum distribution and the expectation value of the stochastic 
fields that sample the Wigner distribution $W$. At large times, Eq. (6) reduces to the simple scaling $n(\mathbf{k} \rightarrow 0$, $t \rightarrow \infty)=2\left(g n_{c}(0) / \gamma\right)^{2} \exp (-\gamma t / \hbar)$.

For the limit of large $k$, we can use the sudden approximation from [22]. We used the Bogoliubov transformation to derive the expectation values for the Bogoliubov excitations at $t=0$. Neglecting the time dependence of the polariton density, the expectation values for the polariton momentum distribution can be expressed in terms of those for the Bogoliubov excitations [23]. Of the four terms, two represent the average value, whereas the other two are terms oscillating in time. These last two terms we neglect, and the time dependence due to the losses is included by an exponential decay. As a result we find for the momentum distribution

$$
n(k, t)=\frac{1}{2}\left[\frac{g n_{c}(0)}{\hbar \omega_{B}(k)}\right]^{2} e^{-\gamma t / \hbar},
$$

where $\hbar \omega_{B}(k)=\sqrt{\epsilon(k)\left[\epsilon(k)+2 g n_{c}(t)\right]}$. The wave number $k_{*}$ where the crossover between the small (6) and large (7) momentum behavior takes place can be found by equating both expressions. In the limit $g n_{c}(0) \ll \gamma$, this $k_{*}$ tends to zero and the expression (7) becomes accurate down to very small wave vectors. In the more interesting limit where $g n_{c}(0) \gg \gamma$, this yields

$$
k_{*}(t)=\frac{\gamma}{2 \hbar} \sqrt{\frac{m}{g n_{c}(0)}}\left[1-e^{-\gamma t / \hbar}\left(\frac{\gamma t}{\hbar}+1\right)\right]^{-1 / 2},
$$

where $m$ is the polariton mass.

For the behavior of the momentum distribution around $k_{*}(t)$, we have not found an analytic formula, but the Bogoliubov dynamics (5) were solved numerically instead. To this purpose, we wrote down a formal solution to Eq. (5) in terms of a Green's function that describes the evolution over the time interval of interest [23]. In the computation this time interval is discretized. The results obtained with this numerical scheme are presented in Fig. 2 by the solid blue lines. We have rescaled them to the condensate density in order to cancel the trivial exponential decay. The black dashed horizontal line represents the prediction (6) for $t \rightarrow \infty$, which matches perfectly with the numerical result at late times. The red dotted line represents expression (7), which is seen to match very well for sufficiently large momenta, justifying the sudden approximation in this regime. For the smaller interaction strength [Fig. 2(a)], the momentum distribution decays as $k^{-4}$, where for the larger interaction strength [Fig. 2(b)], its decay is dominated by the intermediate $k^{-2}$ region, although for larger momenta the momentum distribution also decays as $k^{-4}$. This $k^{-4}$ behavior is a general result derived in several systems with repulsive contact interactions. It was found, e.g., in a Lieb-Liniger gas for all values of the interaction strength
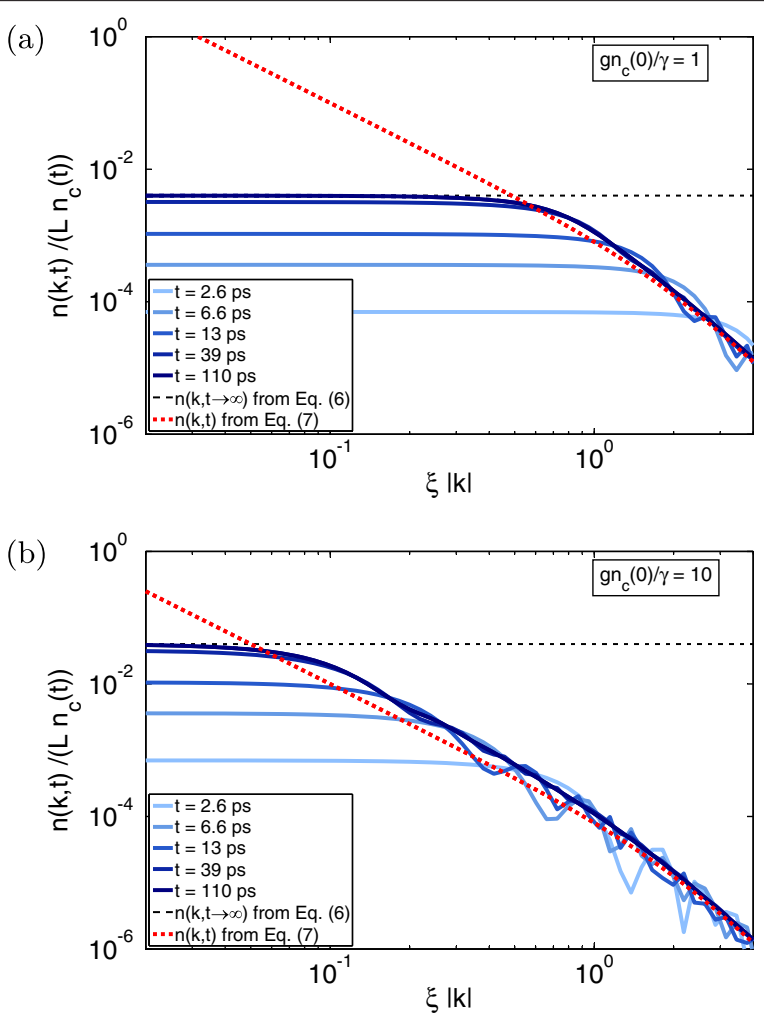

FIG. 2 (color online). The momentum distribution $n(k)$ of the polariton gas (of arbitrary dimensionality) due to the dynamical Casimir effect. The momentum distribution is normalized by the number of particles in the condensate and given as a function of the unitless parameter $\xi|k|$ with $\xi=\hbar / \sqrt{m g n_{c}(0)}$ the initial healing length. The full lines show the numerical result for various times. The black dashed line is the prediction (6) for small $\mathbf{k}$ at large times; the red dotted line represents the asymptotic result (7). We have chosen $g=0.01 \mu \mathrm{mmeV}$, $\gamma=0.05 \mathrm{meV}, L=100 \mu \mathrm{m}, \hbar=1, m=1$. (a) $g n_{c}(0) / \gamma=1$. (b) $g n_{c}(0) / \gamma=10$.

[24] and in a harmonically confined Tonks-Girardeau gas at zero [25] and finite temperature [26].

The total number of particles produced through the dynamical Casimir effect can now be computed by integrating the momentum distribution $n(k, t)$ over $k$. The result for the total number of particles will depend on the dimensionality of the system, because of the integration. Here, we will evaluate the particle production for a onedimensional polariton wire, where quantum fluctuations are most important due to the high density of states at low momenta. The ratio of particles in nonzero momentum states to the condensate density $n_{c}(t)$ can be written as

$$
\frac{\delta n(t)}{n_{c}(t)}=\lim _{k^{\prime} \rightarrow 0} \frac{n\left(k^{\prime}, t\right)}{2 \pi n_{c}(t)} \int d k \frac{n(k, t)}{n\left(k^{\prime}, t\right)}
$$

where we have taken the continuum limit. The integrand in Eq. (9) is approximately one for $k<k_{*}$, where for $k>k_{*}$ it can be computed from Eqs. (6) and (7). Thus, we find that the particle production scales in the limit $g n_{c}(0) \gg \gamma$ as 


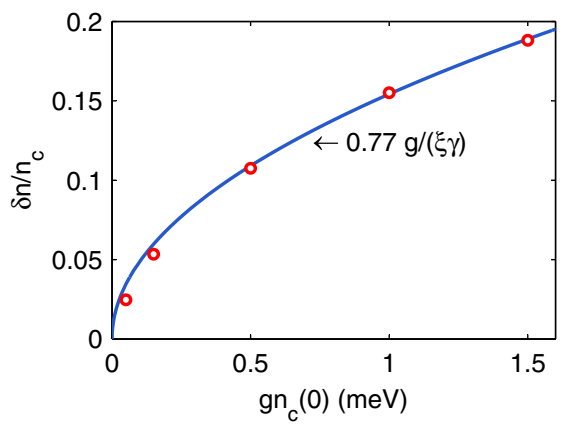

FIG. 3 (color online). The total quantum depletion $\delta n(t) / n_{c}(t)$ of a one-dimensional polariton gas for $t \rightarrow \infty$ as a function of the initial blueshift $g n_{c}(0)$. The circles show the numerical result; the full line is the analytical prediction. We have taken an interaction strength $g=0.01 \mu \mathrm{m} \mathrm{meV}$ and a linewidth $\gamma=0.05 \mathrm{meV}$.

$$
\frac{\delta n(t)}{n_{c}(t)}=C \frac{g^{2} n_{c}(0)}{\gamma^{2}}\left[1-e^{-\gamma t / \hbar}\left(\frac{\gamma t}{\hbar}+1\right)\right] k_{*}(t),
$$

where the constant can be computed numerically from the full momentum distribution: $C \approx 1.5$. At large times, the depletion (10) can be written as $\delta n / n \approx 0.77 \mathrm{~g} /(\xi \gamma)$, where $\xi=\hbar / \sqrt{m g n_{c}(0)}$ is the healing length of the condensate immediately after its creation. This shows that the particle production is proportional to the blockade parameter $U / \gamma$ [19], where $U=g / \xi$ is the interaction energy of two polaritons localized within the condensate healing length. Typical attainable values of the healing length are one micron, so that for an interaction strength $g=$ $0.01 \mathrm{meV} \mu \mathrm{m}$, a linewidth $\gamma=0.05 \mathrm{meV}$ is sufficient to obtain more than $10 \%$ of quantum depletion. Figure 3 compares the analytical estimate (10) with the depletion computed from the full numerical momentum distribution. It is already accurate for moderate values of the parameter $g n_{c}(0) / \gamma \geq 1$. Although large values of this parameter seem attainable with state of the art microcavities [27], this regime can no longer be described by the Bogoliubov approximation, and goes beyond the scope of the present Letter.

Experimentally, the quantum depletion is directly accessible through a measurement of the first-order spatial coherence $g^{(1)}\left(x-x^{\prime}\right)=\left\langle\psi^{\dagger}(x, t) \psi\left(x^{\prime}, t\right)\right\rangle / n_{c}(t)$. The fluctuations that are created because of parametric scattering lead to a drop in the first-order coherence function equal to the quantum depletion: $g^{(1)}(x \rightarrow 0)-g^{(1)}(x \rightarrow \infty)=$ $\delta n / n_{c}$. We restrict the calculations again to a onedimensional wire, as we did for $\delta n / n_{c}$.

The numerical results for the first-order coherence are presented in Fig. 4. Since the gas is assumed to be created by a perfectly coherent laser, no fluctuations are present initially. The correlation length can be estimated from the relation $\ell_{c}(t) \propto 1 / k_{*}(t)$. The red circles in Fig. 4 show this prediction to work well. From this relation, it is seen that at short times the coherence length scales as
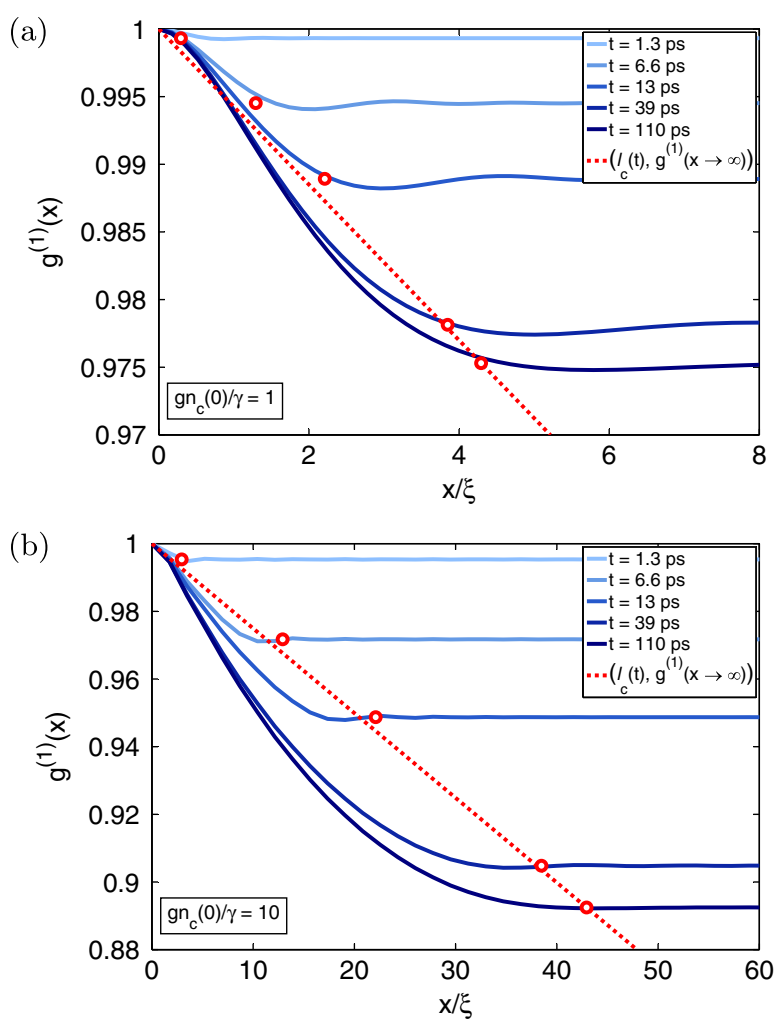

FIG. 4 (color online). The spatial coherence $g^{(1)}(x)$ of a onedimensional polariton gas as a function of time, for the same parameters as the corresponding panels in Fig. 2. The red circles represent the analytical estimate of the correlation length $\ell_{c}(t)=2.1 / k_{*}(t)$. The red dotted line shows the linear relation between the correlation length and the depletion.

$\ell_{c}(t) \propto 2 t \sqrt{g n_{c}(0) / m}$. This is indeed what one expects for fluctuations that spread out at the speed of sound $c=\sqrt{g n_{c}(0) / m}$. At long times, we obtain $\ell_{c}(t \rightarrow \infty)=$ $4.3 \sqrt{g n_{c}(0) / m} \hbar / \gamma$, where the constant 4.3 was determined numerically. This means that the effective propagation time of the fluctuations is the lifetime $\tau=\hbar \gamma^{-1}$; their average speed is proportional to the initial sound velocity. Finally, Fig. 4 depicts an interesting linear relation between the time dependent depletion and the coherence length: $\ell_{c}(t) \propto$ $\delta n(t) / n_{c}(t)$. By combining Eqs. (8) and (10), this relation is readily verified analytically. In experiments, fluctuations during a laser pulse are likely to occur. However, these do not result in any additional spatial decoherence, but result in an averaging of the quantum depletion.

The dynamical Casimir generation of Bogoliubov excitations that we have studied in this Letter is only the first stage of the dynamics. For long polariton lifetimes, this opens the prospect to explore the physics of the (pre) thermalization of 1D quantum systems [28] in a new setting.

The authors acknowledge financial support from the FWO Odysseus program and stimulating discussions with I. Carusotto. 
*selma.koghee@uantwerpen.be

[1] G. T. Moore, J. Math. Phys. (N.Y.) 11, 2679 (1970).

[2] S. A. Fulling and P. C. W. Davies, Proc. R. Soc. A 348, 393 (1976).

[3] N. Birrell and P. Davies, Quantum Fields in Curved Space (Cambridge University Press, Cambridge, England, 1984).

[4] P. O. Fedichev and U. R. Fischer, Phys. Rev. A 69, 033602 (2004).

[5] P. Jain, S. Weinfurtner, M. Visser, and C. W. Gardiner, Phys. Rev. A 76, 033616 (2007).

[6] C. M. Wilson, G. Johansson, A. Pourkabirian, M. Simoen, J. R. Johansson, T. Duty, F. Nori, and P. Delsing, Nature (London) 479, 376 (2011).

[7] P. Lähteenmäki, G. S. Paraoanu, J. Hassel, and P. J. Hakonen, Proc. Natl. Acad. Sci. U.S.A. 110, 4234 (2013).

[8] L. J. Garay, J. R. Anglin, J. I. Cirac, and P. Zoller, Phys. Rev. Lett. 85, 4643 (2000).

[9] R. Balbinot, A. Fabbri, S. Fagnocchi, A. Recati, and I. Carusotto, Phys. Rev. A 78, 021603 (2008).

[10] O. Lahav, A. Itah, A. Blumkin, C. Gordon, S. Rinott, A. Zayats, and J. Steinhauer, Phys. Rev. Lett. 105, 240401 (2010).

[11] J.-C. Jaskula, G. B. Partridge, M. Bonneau, R. Lopes, J. Ruaudel, D. Boiron, and C. I. Westbrook, Phys. Rev. Lett. 109, 220401 (2012).

[12] I. Carusotto and C. Ciuti, Rev. Mod. Phys. 85, 299 (2013).

[13] A. Amo, J. Lefrère, S. Pigeon, C. Adrados, C. Ciuti, I. Carusotto, R. Houdré, E. Giacobino, and A. Bramati, Nat. Phys. 5, 805 (2009).

[14] D. D. Solnyshkov, H. Flayac, and G. Malpuech, Phys. Rev. B 84, 233405 (2011).

[15] D. Gerace and I. Carusotto, Phys. Rev. B 86, 144505 (2012).
[16] V. Kohnle, Y. Léger, M. Wouters, M. Richard, M. T. Portella-Oberli, and B. Deveaud, Phys. Rev. B 86, 064508 (2012).

[17] J. Adamek, X. Busch, and R. Parentani, Phys. Rev. D 87, 124039 (2013).

[18] E. Wertz, L. Ferrier, D. D. Solnyshkov, R. Johne, D. Sanvitto, A. Lemaître, I. Sagnes, R. Grousson, A. V. Kavokin, P. Senellart, G. Malpuech, and J. Bloch, Nat. Phys. 6, 860 (2010).

[19] A. Verger, C. Ciuti, and I. Carusotto, Phys. Rev. B 73, 193306 (2006).

[20] C. W. Gardiner, Quantum Noise (Springer-Verlag, Berlin, 1991).

[21] P. B. Blakie, A. S. Bradley, M. J. Davis, R. J. Ballagh, and C. W. Gardiner, Adv. Phys. 57, 363 (2008).

[22] I. Carusotto, R. Balbinot, A. Fabbri, and A. Recati, Eur. Phys. J. D 56, 391 (2010).

[23] See Supplemental Material at http://link.aps.org/ supplemental/10.1103/PhysRevLett.112.036406 for more detailed calculations on the large $k$ behavior using the sudden approximation and on the Green's function method which forms the basis of the numerical computations.

[24] M. Olshanii and V. Dunjko, Phys. Rev. Lett. 91, 090401 (2003).

[25] A. Minguzzi, P. Vignolo, and M. P. Tosi, Phys. Lett. A 294, 222 (2002).

[26] P. Vignolo and A. Minguzzi, Phys. Rev. Lett. 110, 020403 (2013).

[27] B. Nelsen, G. Liu, M. Steger, D. W. Snoke, R. Balili, K. West, and L. Pfeiffer, Phys. Rev. X 3, 041015 (2013).

[28] A. Polkovnikov, K. Sengupta, A. Silva, and M. Vengalattore, Rev. Mod. Phys. 83, 863 (2011). 